\title{
ISFET pH-Sensor Sensitivity Extraction Using Conventional MOSFET Simulation Tools
}

\author{
Tarek M. Abdolkader, Abdurrahman G. Alahdal, Ahmed Shaker, and Wael Fikry
}

\begin{abstract}
The Ion-Sensitive Field-Effect Transistor (ISFET) has traditionally been used to measure hydrogen ion concentration ( $\mathrm{pH}$ ) of a solution. Its performance depends mainly on its sensitivity to $\mathrm{pH}$ change of the electrolyte in contact with its gate. This sensitivity is usually calculated by examining the effect of $\mathrm{pH}$ value on the charge and potential distributions above gate insulator, which is translated into a shift in the threshold voltage. In this work, we propose a methodology to extract the sensitivity of ISFET by linking electrolyte charge and potential equations with a device simulation tool to calculate the ISFET's drain current, thus, taking into account the underlying structure's physical properties. Using the proposed methodology, the sensitivity of ISFET is compared for various $\mathrm{pH}$ values and gate-insulator thicknesses searching for the optimum conditions that give the highest sensitivity.
\end{abstract}

Index Terms-ISFET, pH-sensor, biosensors, device simulation.

\section{INTRODUCTION}

Ion-Sensitive Field-Effect Transistor (ISFET) has been used for detection of the ionic activity in an electrolyte solution attached to the gate oxide of a Metal-Oxide-Semiconductor (MOS) structure. One of the most useful parameters to be measured in biochemistry is the hydrogen concentration, or $\mathrm{pH}$. ISFET is one of the leading $\mathrm{pH}$ sensors using semiconductor technologies [1]. It has recently attracted more interest because of many advantages compared to other $\mathrm{pH}$ sensing methods such as litmus papers and glass $\mathrm{pH}$ electrodes [2], [3]. It has a relatively high sensitivity, smaller size, and it can be used at high temperature. Moreover, it suits continuous monitoring, has potential for large-scale integration, and may be fabricated using conventional CMOS process. ISFETs have been adopted in various lab-on-chip and health-care applications [4]. It found use also in agriculture, environmental monitoring, and food industries [2].

Fig. 1 shows a schematic illustration for the ISFET structure. It resembles normal Metal-Oxide-Semiconductor Field-Effect Transistor (MOSFET) structure except that the

Manuscript received September 25, 2014; revised January 10, 2015. Sponsor and financial support acknowledgment: This work was supported by the Deanship of Scientific Research, Umm Al-Qura University, Makkah, Saudi Arabia under Grant 43408011".

Tarek M. Abdolkader and A. G. Alahdal are with the Electrical Engineering Department, Faculty of Engineering, Umm Al-Qura University, Saudi Arabia, on leave from Benha University, Benha, Egypt (e-mail: tmhasan@uqu.edu.sa,agahdal@uqu.edu.sa).

A. Shaker and W. Fikry are with the Engineering Physics Department, Ain Shams University, Egypt (email: ashaker2k@yahoo.com, waelfikry@yahoo.com). metal gate of the latter is replaced by an electrolyte solution to be tested, with a reference metal electrode immersed in this electrolyte. A change in the hydrogen ion concentration of the electrolyte ( $\mathrm{pH}$ change) induces a change in the charge distribution on the surface of the gate insulator and this will in turn change the drain current $I_{\mathrm{D}}$ in the MOSFET [5]. $\mathrm{pH}$ measurement may be done by relating it to the change in drain current $I_{\mathrm{D}}$ at a fixed reference electrode voltage $\left(V_{\text {ref }}\right)$. In this case, the sensitivity is expressed as $I_{\mathrm{D}}$ change per $\mathrm{pH}$ unit change. Alternatively, $\mathrm{pH}$ may be measured by finding the required $V_{\text {ref }}$ shift to maintain fixed $I_{\mathrm{D}}$ value. [2]. Here, it becomes the change in $V_{\text {ref }}$ per $\mathrm{pH}$ unit change.

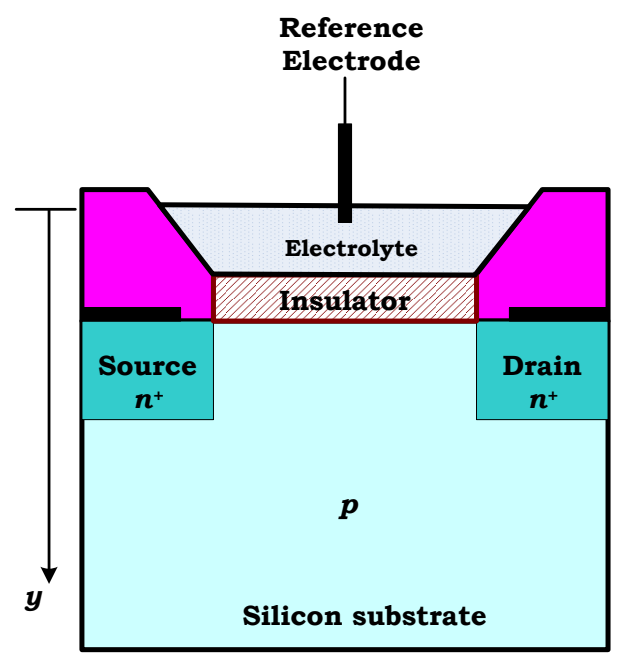

Fig. 1. Schematic diagram of ISFET structure.

As the sensitivity of an ISFET is an important parameter determining its performance, many attempts for modeling it were reported [3], [6]-[8]. All these attempts use an approximate analytical expression to describe the electrostatics of the underlying MOSFET.

Although ISFETs have been fabricated in CMOS for a variety of physical geometries, it has not yet been reported how design dimensions impact sensor characteristics [6]. In this work, we propose a methodology for extracting the sensitivity of the ISFET using full numerical simulation of its underlying MOSFET, and thus, include the effect of MOSFET structure parameters more accurately. The proposed methodology is used to compare the ISFET sensitivity at the whole $\mathrm{pH}$ range, and for various current levels and reference voltage values. In addition, the effect of the gate-insulator thickness is also examined. This helps to optimize the ISFET performance by choosing the most suitable conditions of operation.

Section II reviews ISFET theory depicting its electrostatic equations. Section III presents the new proposed 
methodology. Simulation results are given and discussed in Section IV followed by the conclusion in Section V.

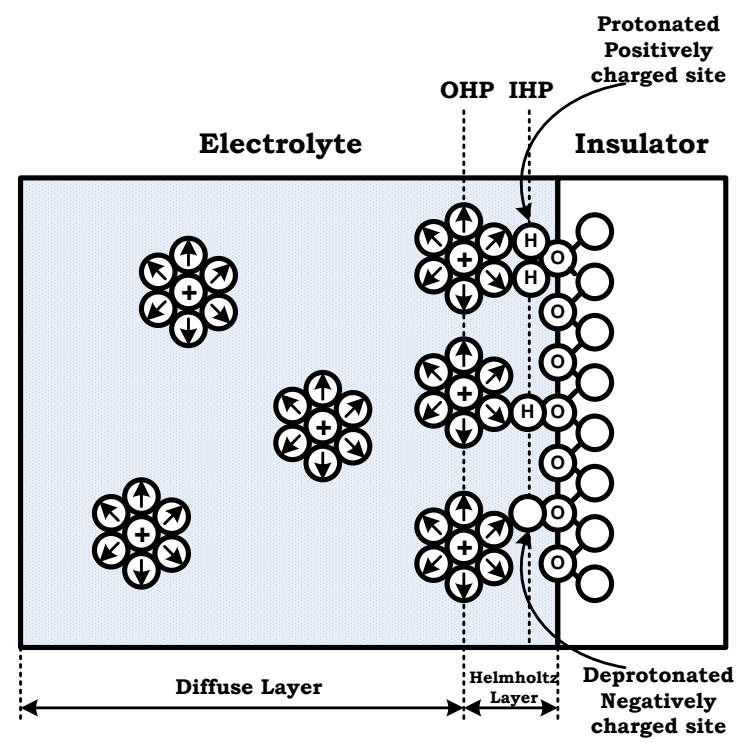

Fig. 2. Binding sites and double electrical layer at electrolyte-insulator interface.

\section{ISFET THEORY}

ISFET operation is explained using site-binding theory [9]. The insulating surface of the ISFET's gate contains hydroxyl groups $(\mathrm{OH})$ that forms binding sites. They can be positively charged (by acquiring $\mathrm{H}^{+}$) or negatively charged (by losing $\mathrm{H}^{+}$) depending on the concentration of the hydrogen ions in the electrolyte. Accordingly, the insulating surface is charged with surface charge density that depends on the solution's $\mathrm{pH}$.

According to Gouy-Chapman-Stern theory [9], electrolyte ions (anions and cations) form three regions ( Fig. 2). The first extends from the Insulator-Electrolyte interface to the Inner Helmholtz Plane (IHP), which is the plane passing through the centers of the specifically adsorbed ions. Next, the region from IHP to the Outer Helmholtz Plane (OHP) that passes through the centers of the hydrated ions at their distance of closest approach to the solid. The third region consists of a diffuse charge region extending from the OHP into the electrolyte bulk, which is referred to as the Gouy-Chapman layer [7].

As shown in Fig. 3(a), the charge distribution along the $y$-direction (the direction normal to the interface) can be divided into three parts: 1) the inversion/depletion charge in the semiconductor channel, $\sigma_{\mathrm{MOS}}, 2$ ) the surface charge on the Insulator-Electrolyte interface resulting from ion adsorbing, $\sigma_{\mathrm{o}}$, and 3) the continuous charge distribution through the diffuse layer, $\sigma_{\mathrm{d}}$. The corresponding potential distribution is depicted in Fig. 3(b), in which, $V_{\text {ref }}, \psi_{\mathrm{d}}, \psi_{\mathrm{o}}$, and $\psi_{\mathrm{s}}$ are the electric potentials at the reference gate electrode, the edge of diffuse layer (OHP), the electrolyte-insulator interface, and the semiconductor-insulator interface, respectively.

Applying Gauss Law using a Gaussian surface which encompasses the whole structure, we can write [5], [8],

$$
\sigma_{d}+\sigma_{o}+\sigma_{M O S}=0
$$


Fig. 3. (a) Surface charge density and (b) potential distribution in an Electrolyte-Insulator-Semiconductor (EIS) system along y-direction (the direction normal to the interface).

The first term in the above equation, $\sigma_{d}$, can be related to $\psi_{d}$ and $\psi_{o}$ using a Gaussian surface that encompasses the diffuse layer charge and passing through the Helmholtz layer

$$
\sigma_{d}=C_{h}\left(\psi_{d}-\psi_{o}\right)
$$

where $C_{h}$ is the capacitance of the Helmholtz layer.

In addition, solving Poisson equation in the diffuse layer results in an expression for $\sigma_{d}$ in terms of $\psi_{d}$ which is given as [8],

$$
\sigma_{d}=\sqrt{8 \varepsilon_{o} \varepsilon_{w} k T c_{0}} \sinh \left(\frac{q\left(V_{r e f}-\psi_{d}\right)}{2 k T}\right)
$$

where $\varepsilon_{w}$ is dielectric constant of water, $c_{0}$ is the solution ion concentration.

Second, the surface charge density of the binding sites on the insulator interface, $\sigma_{0}$, is given by [5], [8],

$$
\begin{aligned}
\sigma_{o}= & q N_{s i l}\left(\frac{\left[\mathbf{H}^{+}\right]_{s}^{2}-K_{+} K_{-}}{\left[\mathbf{H}^{+}\right]_{s}^{2}+K_{+}\left[\mathbf{H}^{+}\right]_{s}+K_{+} K_{-}}\right) \\
& +q N_{n i t}\left(\frac{\left[\mathbf{H}^{+}\right]_{s}}{\left[\mathbf{H}^{+}\right]_{s}+K_{N+}}\right)
\end{aligned}
$$

where $N_{\text {sil }}$ and $N_{\text {nit }}$ are the number of silanol sites and primary amine sites per unit area, respectively; $K_{+}, K_{-}$, and $K_{\mathrm{N}+}$ are the dissociation constants for positively charged silanol sites, negatively charged silanol sites, and positively charged amine sites, respectively; $\left[\mathrm{H}^{+}\right]_{s}$ is the concentration of protons at the insulator surface. 
It should be noted that $\left[\mathrm{H}^{+}\right]_{s}$ is related to the concentration of protons inside the electrolyte bulk, $\left[\mathrm{H}^{+}\right]_{b}$ by [8]

$$
\left[\mathbf{H}^{+}\right]_{s}=\left[\mathbf{H}^{+}\right]_{b} \exp \left(\frac{q\left(V_{r e f}-\psi_{o}\right)}{k T}\right)
$$

where the $\mathrm{pH}$ of the solution is $-\log \left(\left[\mathrm{H}^{+}\right]_{b}\right)$.

The third type of charge density, $\sigma_{\mathrm{MOS}}$, is related to $\psi_{\mathrm{o}}$ and $\psi_{\mathrm{s}}$ through Gauss law

$$
\sigma_{M O S}=C_{i n s}\left(\psi_{s}-\psi_{o}\right)
$$

where $C_{\text {ins }}$ is the capacitance of the insulator.

Another equation that is needed to solve the system is the relation representing the effect of underlying MOSFET structure on the surface potential and charge density [8]

$$
\begin{aligned}
\sigma_{M O S}= & \pm \sqrt{2 \varepsilon_{o} \varepsilon_{S i} k T p_{0}} \\
& \left\{\begin{array}{l}
\left(\frac{q \psi_{s}}{k T}-1+\exp \left(-\frac{q \psi_{s}}{k T}\right)\right) \\
+\frac{n_{o}}{p_{o}}\left(-\frac{q \psi_{s}}{k T}-1+\exp \left(\frac{q \psi_{s}}{k T}\right)\right)
\end{array}\right\}
\end{aligned}
$$

where $\varepsilon_{S i}$ is dielectric constant of silicon; $n_{o}$ and $p_{o}$ are the equilibrium electron and hole concentrations within silicon.

The seven equations (1)-(7) has seven unknowns: three charge densities, three surface potentials, and $\left[\mathrm{H}^{+}\right]_{\mathrm{s}}$, so, they can be solved simultaneously to get all potentials. Once $\psi_{\mathrm{o}}$ is known, the shift in the threshold voltage is determined by $\left(V_{\text {ref }}-\psi_{\mathrm{o}}\right)[9]$, so, the new threshold voltage will be,

$$
V_{T}^{\prime}=V_{T}-\left(V_{r e f}-\psi_{o}\right)
$$

where $V_{\mathrm{T}}$ is the threshold voltage of the basic MOSFET structure. Once $V_{T}^{\prime}$ is known, the drain to source current can be found analytically using any of the standard MOSFET models [10]. For example, simple charge control model can be used to get the drain current [3],

$$
\begin{aligned}
I_{D}= & \frac{W \mu_{n} C_{i n s}}{L} \times \\
& \left\{\begin{array}{ll}
\left(V_{G}-V_{T}^{\prime}-V_{D} / 2\right) V_{D} & V_{D} \leq\left(V_{G}-V_{T}^{\prime}\right) \\
\left(V_{G}-V_{T}^{\prime}\right)^{2} / 2 & V_{D}>\left(V_{G}-V_{T}^{\prime}\right)
\end{array}\right\}
\end{aligned}
$$

Two ways can be used to express ISFET sensitivity: the first is using $S_{1}$ defined as the absolute value of change in $I_{\mathrm{D}}$ per unit change in $\mathrm{pH}$ with $V_{\text {ref }}$ fixed, so

$$
S_{1}=\left|\frac{\delta I_{D}}{\delta p H}\right|_{V_{\text {ref }} \text { (constant) }}
$$

The second is using $S_{2}$ defined as the absolute value of change in $V_{\text {ref }}$ per unit change in $\mathrm{pH}$ that is required to maintain $I_{\mathrm{D}}$ fixed

$$
S_{2}=\left|\frac{\delta V_{r e f}}{\delta p H}\right|_{I_{D} \text { (constant) }}
$$

Once the drain current $I_{\mathrm{D}}$ is calculated for a certain reference voltage $V_{\text {ref }}$ at a certain $\mathrm{pH}, I_{\mathrm{D}}$ is found again for a neighboring $\mathrm{pH}$ value, and $S_{1}$ is found from (10). Similar procedure can be used for $S_{2}$.

\section{Proposed Simulation Method}

Calculation of ISFET sensitivity presented in the last section uses approximate modeling equations to describe MOSFET electrical properties. This is represented by equations (6)-(9) which are based on simplifying assumptions to reach closed-form analytical formulae. On the other hand, numerical device simulation of MOSFET represents device physics more accurately and takes into account process effects more deeply. So, in this work, we replace these modeling equations by linking ISFET charge and potential equations (1)-(5) to the numerical device simulation tool, Silvaco [11] in order to find a complete solution of the system. The methodology is shown in the flow chart in Fig. 4 and explained hereinafter.

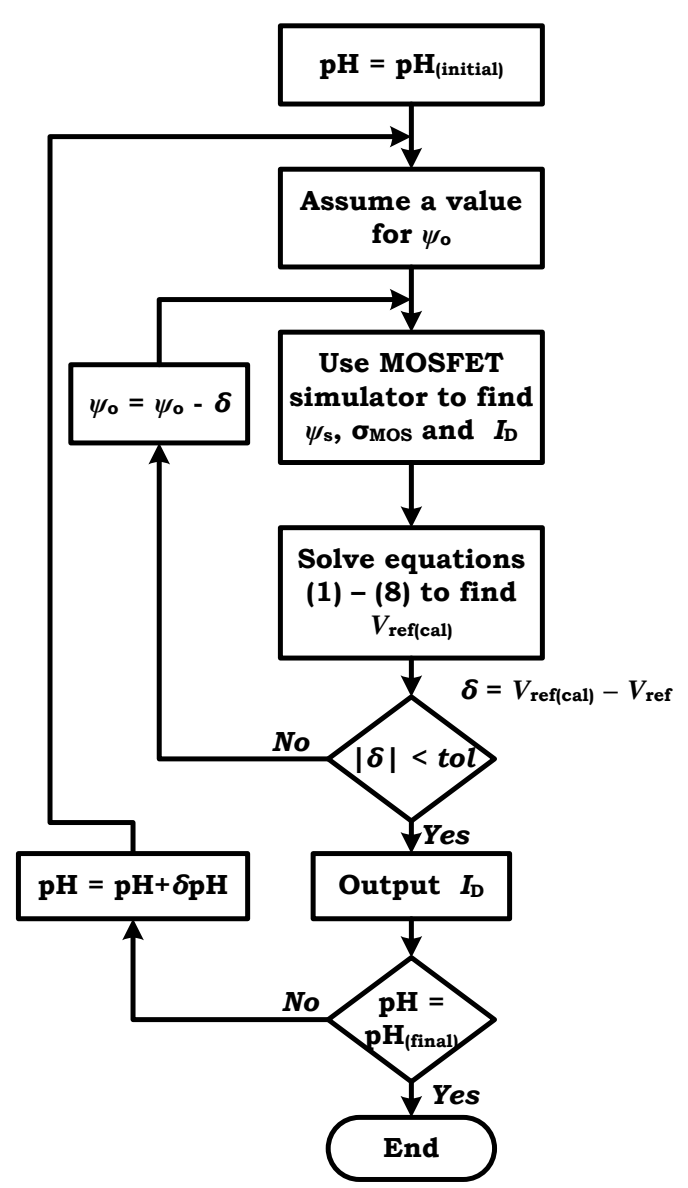

Fig. 4. Flow chart for the methodology proposed for the extraction of ISFET sensitivity.

At the beginning, for a certain $\mathrm{pH}$ of the solution, and for a 
certain reference electrode voltage $V_{\text {ref }}$, a value of $\psi_{\mathrm{o}}$ is assumed (roughly half of the reference electrode voltage, $\left.V_{\text {ref }}\right)$. Then, a device simulation using Silvaco tools is performed for a conventional metal-gated MOSFET with its gate bias equals $\psi_{\mathrm{o}}$. From the simulation results we can calculate $\psi_{\mathrm{s}}, \sigma_{\mathrm{MOS}}$, and $I_{\mathrm{D}}$. Now, equations (1)-(5) can be solved for the unknowns: $\sigma_{\mathrm{o}}, \sigma_{\mathrm{d}}, \psi_{\mathrm{d}}, V_{\text {ref }}$, and $\left[\mathrm{H}^{+}\right]_{\mathrm{s}}$ and, consequently, a new value of $V_{\text {ref }}$ is found which we call it $V_{\text {ref(cal). }}$. The calculated $V_{\text {ref(cal) }}$ is compared to the real value of the reference electrode voltage, $V_{\text {ref }}$, and the difference is subtracted from the firstly assumed $\psi_{0}$. Now, the new updated value of $\psi_{\mathrm{o}}$ is used to perform a new simulation run. The whole process is repeated until the difference between the calculated and real values of $V_{\text {ref }}$ is within a certain tolerance. After reaching a consistent solution for both $\psi_{\mathrm{o}}$ and $V_{\text {ref }}$, the drain current output from simulation is stored for the corresponding value of $\mathrm{pH}$ and $V_{\text {ref. }}$.

If, conversely, the drain current $I_{\mathrm{D}}$ is predetermined and it is required to find the corresponding reference voltage $V_{\text {ref }}$ that produces this current at certain $\mathrm{pH}$, simulation runs are repeated with $\psi_{\mathrm{o}}$ incremented until it produces the predetermined current, then, equations (1)-(5) are solved only once to get $V_{\text {ref }}$.

All calculations are repeated for different $\mathrm{pH}$ values. Once $I_{\mathrm{D}}$ and $V_{\text {ref }}$ are known for each value of $\mathrm{pH},(10)$ and (11) can be used to calculate ISFET sensitivities $S_{1}$ and $S_{2}$.

\section{RESUlTS AND DISCUSSION}

The method described in the last section was used to study the effect of various properties of ISFET $\mathrm{pH}$ sensor on the output current and on the two types of sensitivity defined in (10) and (11). The drain to source voltage is set to $0.2 \mathrm{~V}$. The other parameter values used for simulation are shown in Table I, in which, $N_{\mathrm{A}}$ is the substrate doping, $L$ is the gate length, and $W$ is the device width.

TABLE I: THE PARAMETER VALUES USED FOR SIMULATION

\begin{tabular}{cc|cc}
\hline \hline$\varepsilon_{w}$ & 78.3 & $c_{0}$ & $2 \times 10^{15} \mathrm{~cm}^{-3}$ \\
\hline$K_{+}$ & $15.8 \mathrm{Mol} / \mathrm{L}$ & $T$ & $300 \mathrm{~K}$ \\
\hline$K_{-}$ & $63.1 \times 10^{-9} \mathrm{Mol} / \mathrm{L}$ & $N_{\mathrm{A}}$ & $1 \times 10^{15} \mathrm{~cm}^{-3}$ \\
\hline$N_{\text {sil }}$ & $5.0 \times 10^{14} \mathrm{~cm}^{-2}$ & $L$ & $1.3 \mu \mathrm{m}$ \\
\hline$C_{h}$ & $20 \times 10^{-6} \mathrm{~F} / \mathrm{cm}^{2}$ & $W$ & $1.0 \mu \mathrm{m}$ \\
\hline \hline
\end{tabular}

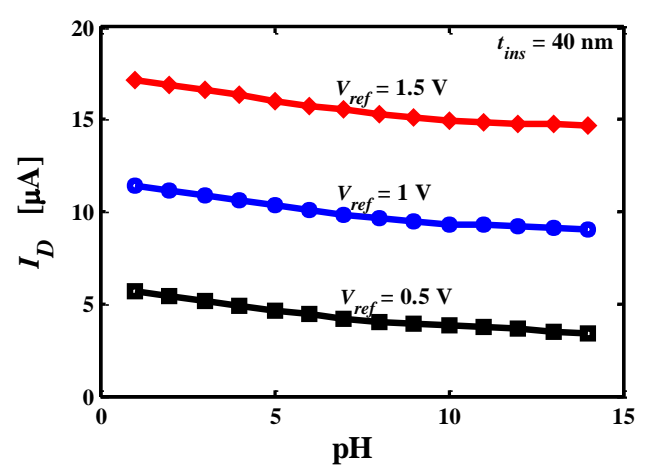

Fig. 5. Drain current variation with $\mathrm{pH}$ value for an ISFET with gate insulator thickness $t_{\text {ins }}=40 \mathrm{~nm}$ at three different values of reference electrode voltage, $V_{\text {ref }}=0.5,1$, and $1.5 \mathrm{~V}$.

Fig. 5 shows the variation of the drain current versus $\mathrm{pH}$ value for $\mathrm{SiO}_{2}$ gate-insulator of thickness $t_{\text {ins }}=40 \mathrm{~nm}$ at three values of reference electrode voltage $V_{\text {ref }}, 0.5,1$, and $1.5 \mathrm{~V}$. The change in the drain current is seen to be relatively larger for small $\mathrm{pH}$ compared to high $\mathrm{pH}$ range. On the other hand, in Fig. 6, $V_{\text {ref }}$ is plotted against $\mathrm{pH}$ at three current levels of 10 , 13 , and $15 \mu \mathrm{A}$. The change in $V_{\text {ref }}$ is also larger at low $\mathrm{pH}$ values. More quantitatively, in Fig. 7, the sensitivity $S_{1}$ is plotted versus $\mathrm{pH}$ for the same device at $V_{\text {ref }},=0.5,1$, and 1.5 V. Although the sensitivity generally decreases with increasing $\mathrm{pH}$, however, it increases again for large $\mathrm{pH}$,

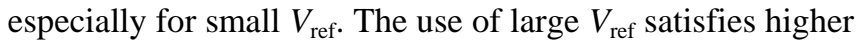
maximum sensitivity (attained at very low $\mathrm{pH}$ ), but on the other hand, the use of small $V_{\text {ref }}$ gives better sensitivity at very high $\mathrm{pH}$. The same conclusion can be extracted from Fig. 8, in which, the other type of sensitivity $S_{2}$ is plotted versus $\mathrm{pH}$ for three level of drain currents, $I_{\mathrm{D}}=3,10$, and $17 \mu \mathrm{A}$. Another design factor, which is the level of output drain current, can be viewed from Fig. 8. Small current levels are suitable for large $\mathrm{pH}$ solutions while large current levels are better for small $\mathrm{pH}$ solutions.

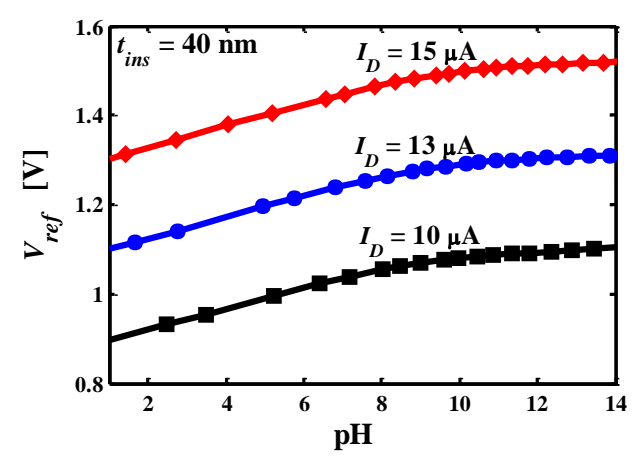

Fig. 6. Reference electrode voltage versus $\mathrm{pH}$ for an ISFET with gate insulator thickness $t_{\text {ins }}=40 \mathrm{~nm}$ at three fixed current levels, $I_{\mathrm{D}}=10,13$, and $15 \mu \mathrm{A}$.

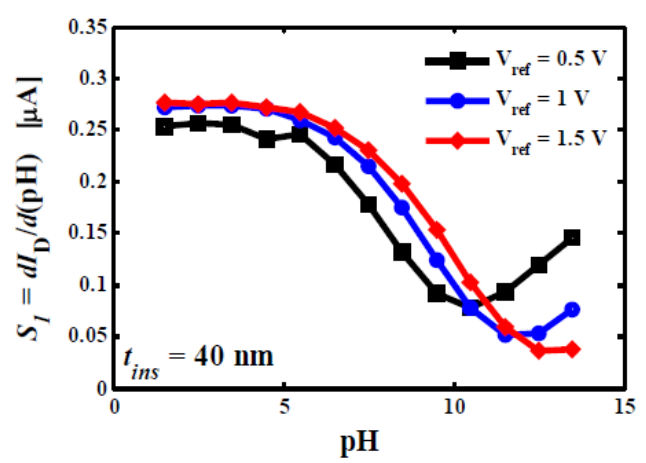

Fig. 7. ISFET sensitivity, $S 1$ versus $\mathrm{pH}$ value at three values of $V$ ref, $0.5,1$ and $1.5 \mathrm{~V}$ for a device of gate-insulator thickness $t$ ins $=40 \mathrm{~nm}$. Sensitivity deteriorates for large values of $\mathrm{pH}$.

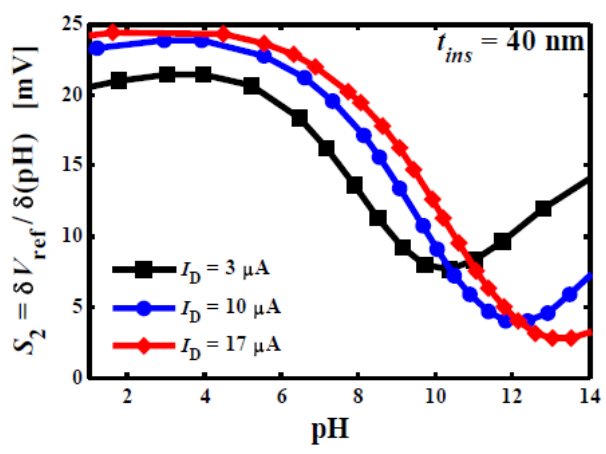

Fig. 8. ISFET sensitivity, $S 2$ versus $\mathrm{pH}$ value at three values of $I \mathrm{D}, 3,10$ and $17 \mu \mathrm{A}$ for a device of gate-insulator thickness $t$ ins $=40 \mathrm{~nm}$. 


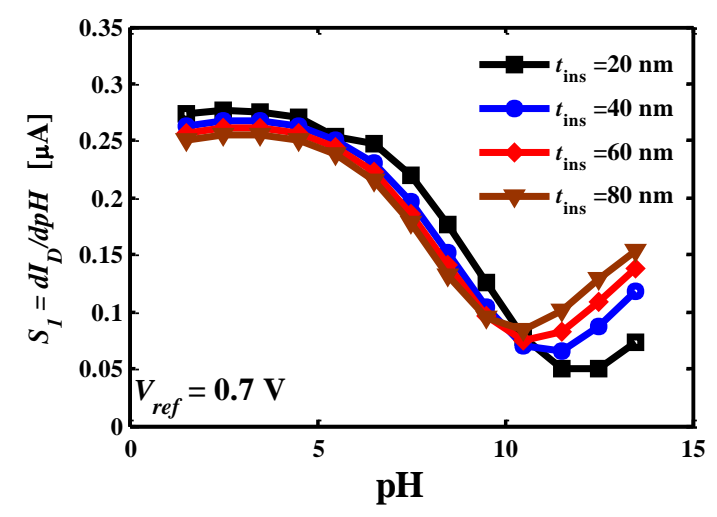

Fig. 9. ISFET sensitivity, $S_{1}$ versus $\mathrm{pH}$ value at $V_{\text {ref }}=0.7 \mathrm{~V}$ and for four gate-insulator thicknesses $t_{\text {ins }}=20,40,60$, and $80 \mathrm{~nm}$.

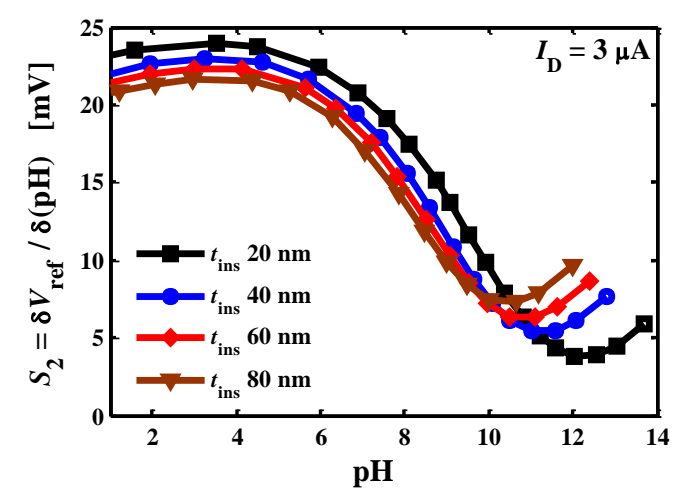

Fig. 10. ISFET sensitivity, $S_{2}$ versus $\mathrm{pH}$ value at $I_{\mathrm{D}}=3 \mathrm{~A}$ and for four gate-insulator thicknesses $t_{\text {ins }}=20,40,60$, and $80 \mathrm{~nm}$.

Regarding the effect of gate-insulator thickness, Fig. 9 and 10 shows $S_{1}$ at $V_{\text {ref }}=0.7 \mathrm{~V}$, and $S_{2}$ at $I_{\mathrm{D}}=3 \mu \mathrm{A}$, versus $\mathrm{pH}$ for four values of gate-insulator, $t_{\text {ins }}=20,40,60,80 \mathrm{~nm}$. It should be noted that increasing $t_{\text {ins }}$ has two conflicting effects, the first is to lower the sensitivity at low $\mathrm{pH}(<10)$, and the other is to increase the sensitivity at high $\mathrm{pH}(>10)$. On the average, increasing $t_{\text {ins }}$ will improve the sensitivity of the device as a whole and guarantees a higher lower limit on the whole $\mathrm{pH}$ range. This will be on the expense of the decrease of output drain current.

\section{CONCLUSION}

A new methodology was proposed to extract the sensitivity of ISFET pH sensor. The methodology links charge and potential equations in the tested solution with a device simulation tool to account for MOSFET electrical properties. An initial guess for the potential at the insulator surface is assumed, then, simulation is performed to find the corresponding value of the external reference electrode voltage. The process is repeated until the calculated value is consistent with the real applied reference voltage. Various effects on ISFET sensitivity were investigated such as drain current level, reference voltage, $\mathrm{pH}$ range, and gate insulator thickness. It is found that the sensitivity is generally higher for low $\mathrm{pH}$ values. It is also found that working at low current levels and larger gate insulator thicknesses decreases the maximum sensitivity (at low $\mathrm{pH}$ ), but on the other hand it gives the advantage of increasing the minimum sensitivity (at high $\mathrm{pH}$ ).

\section{ACKNOWLEDGMENT}

The authors would like to thank Deanship of Scientific Research at Umm Al-Qura University for the financial support of this project under the grant number 43408011 .

\section{REFERENCES}

[1] H. Nakazawa, R. Otake, M. Futagawa, F. Dasai, M. Ishida, and K Sawada, "High-sensitivity charge-transfer-type ph sensor with quasi-signal removal structure," IEEE Transactions on Electron Devices, vol. 61, pp. 136-140, 2014

[2] U. Hashim, C. S. Weng, and L. W. Wen, "Fabrication of silicon nitride ion sensitive field-effect transistor (ISFET)," in Proc. Micro and 2013 IEEE Regional Symposium on Nanoelectronics (RSM), 2013, pp. 204-207.

[3] J. Dutta, "Modeling ion sensitive field effect transistors for biosensor applications," International Journal of Advanced Research in Engineering and Technology (IJARET), vol. 1, pp. 38-57, June 2010.

[4] Y. Hu and P. Georgiou, "A robust ISFET $\mathrm{pH}$-measuring front-end for chemical reaction monitoring," IEEE Transactions on Biomedical Circuits and Systems, vol. 8, issue 2, pp. 1-11, 2014.

[5] A. V. Surmalyan, "Surface potential behavior in ISFET based bio-(chemical) sensors," Armenian Journal of Physics, vol. 5, pp. 194-202, December 2012.

[6] Y. Liu, P. Georgiou, T. Prodromakis, T. G. Constandinou, and C. Toumazou, "An extended CMOS ISFET model incorporating the physical design geometry and the effects on performance and offset variation," IEEE Transactions on Electron Devices, vol. 58, pp. 4414-4422, 2011.

[7] W. M. Siu and R. S. C. Cobbold, "Basic properties of the electrolyte- $\mathrm{SiO}_{2}-\mathrm{Si}$ system: Physical and theoretical aspects," IEEE Transactions on Electron Devices, vol. 26, pp. 1805-1815, 1979.

[8] M. Grattarola, G. Massobrio, and S. Martinoia, "Modeling H+-sensitive FETs with SPICE," IEEE Transactions on Electron Devices, vol. 39, pp. 813-819, 1992.

[9] J. C. Dutta, "Ion sensitive field effect transistor for applications in bioelectronic sensors: A research review," in Proc. 2012 2nd National Conference on Computational Intelligence and Signal Processing (CISP), 2012, pp. 185-191.

[10] T. Ytterdal, Y. Cheng, and T. A. Fjeldly, Device Modeling for Analog and RF CMOS Circuit Design, John Wiley \& Sons Ltd, 2003.

[11] Silvaco. Technology Computer Aided Design (TCAD) software. [Online]. Available: http://www.silvaco.com/

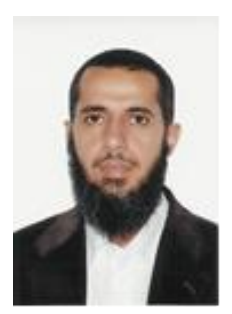

Tarek M. Abdolkader was born in Cairo, Egypt, in 1970. He received two B.S. degrees one in electrical engineering (electronics and communications) from the faculty of engineering, and the other in physics from the faculty of science, both from Ain-Shams University, Cairo, Egypt, in 1992 and 1996, respectively. He received M.S. and Ph.D. degrees in engineering physics from the faculty of Engineering, Ain-Shams University, Cairo, Egypt, in 2001 and 2005, respectively. He joined Faculty of Engineering, Benha University as an assistant professor in 2005 and worked as a post-doctoral research associate in the School of Electrical and Computer Engineering, Purdue University, USA between 2009 and 2010. He is currently in a leave with Electrical Engineering Department, Faculty of Engineering, Umm Al-Qura University, Holy Makka, Saudi Arabia. His research interests are modeling and simulation of Nano-electronic devices including MOSFETs, CNFETs, and ISFETs.
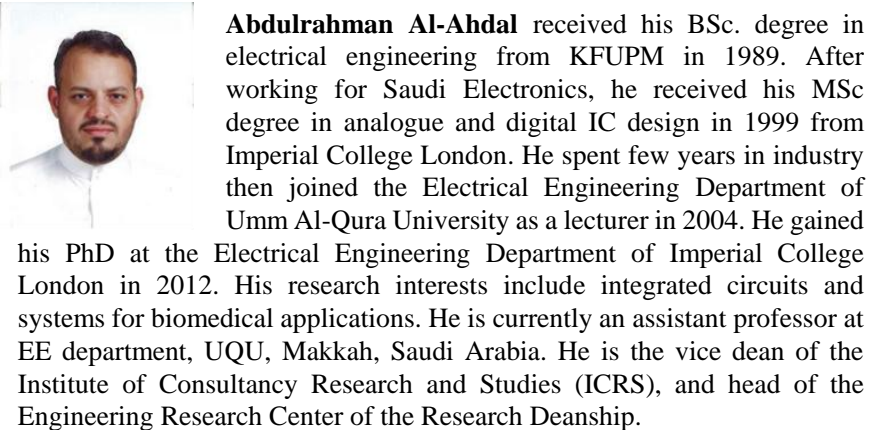
his $\mathrm{PhD}$ at the Electrical Engineering Department of Imperial College London in 2012. His research interests include integrated circuits and systems for biomedical applications. He is currently an assistant professor at EE department, UQU, Makkah, Saudi Arabia. He is the vice dean of the Institute of Consultancy Research and Studies (ICRS), and head of the Engineering Research Center of the Research Deanship. 


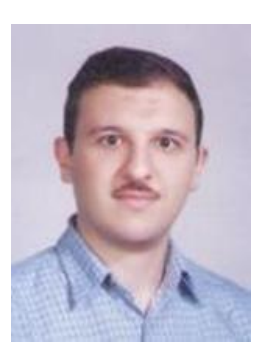

Ahmed Shaker received B.S. degree in electronics and communications from Electrical Engineering Department at Faculty of Engineering, Ain Shams University, Cairo, Egypt, in 1996. He received M.S. and Ph.D. from Engineering Physics Department, Faculty of Engineering, Ain Shams University, Cairo, Egypt, in 2003 and 2010, respectively. He is currently an assistant professor, Engineering Physics \& Mathematics Department Faculty of Engineering, Ain Shams University, Cairo, Egypt. His fields of research are semiconductor physics, simulation and modeling of semiconductor power devices, nanoscale devices, and photovoltaics.



Wael Fikry was born in Cairo, Egypt in 1962. He received the B.S. degree in electrical engineering from Ain Shams University, Faculty of Engineering, Cairo, Egypt in 1984 and the M.S. and $\mathrm{Ph}$.D. degrees in engineering physics from the same university in 1989 and 1994 respectively. He is currently a professor of solid state electronics in the Engineering Physics Department. His research interests include VLSI MOSFET, SOI, Multigate devices characterization and modeling, solar cells and silicon-electrochemical cells. 